\title{
DNA methylation patterns in peripheral blood mononuclear cells from Holstein cattle with variable milk yield
}

\author{
Chad D Dechow *iD and Wan-Sheng Liu
}

\begin{abstract}
Background: Milk yield for Holstein cows has doubled over five decades due to genetic selection and changes to management, but the molecular mechanisms that facilitated this increase are mostly unknown. Epigenetic modifications to the cattle genome are a plausible molecular mechanism to cause variation in milk yield and our objective was to describe genome-wide DNA methylation patterns in peripheral blood mononuclear cells (PBMC) from mature Holstein dairy cows with variable milk yield.

Results: Whole genome MeDIP-seq was performed following DNA extraction from PBMC of 6 lactating dairy cows from 4 different herds that varied in milk yield from 13,556 kg to 23,105 kg per 305 day lactation. We describe methylation across the genome and for 13,677 protein coding genes. Repetitive element reads were primarily mapped to satellite (36.4\%), SINE (29.1\%), and LINE (23.7\%) regions and the majority (78.4\%) of CpG sites were sequenced at least once. DNA methylation was generally low upstream of genes with the nadir occurring $95 \mathrm{bp}$ prior to the transcription start site (TSS). Methylation was lower in the first exon than in later exons, was highest for introns near the intron-exon junctions, and declined downstream as the distance from the gene increased. We identified 72 differentially methylated regions (DMR) between high milk yield cows and their control, and 252 DMR across herd environments.
\end{abstract}

Conclusions: This reference methylome for cattle with extreme variation in milk yield phenotype provides a resource to more fully evaluate relationships between DNA methylation and phenotype in populations subject to selection. The detection of DMR in cows of varying milk yield suggests potential to exploit epigenetic variation in cattle improvement programs.

Keywords: Epigenetics, Selection, Bos taurus, MeDIP-seq, DNA methylation

\section{Background}

Molecular mechanisms that confer phenotypic diversity and that facilitate selective change include DNA methylation [1]. Indeed, genetic selection may act partly through altered epigenetic profiles as DNA sequence variation is reported to cause shifts in DNA methylation [2].

Holstein (Bos taurus) cattle are the most numerous of dairy cattle breeds and produce the largest volumes of milk, fat and protein [3]. Selection for yield in Holstein cattle has increased genetic merit for milk production by $3713 \mathrm{~kg}$, a $\sim 59 \%$ increase, since 1960 [4]. Changes to

\footnotetext{
* Correspondence: cdd1@psu.edu

Department of Animal Science, College of Agricultural Sciences, The

Pennsylvania State University, 324 Henning Building, University Park, State

College, PA 16802, USA
}

(c) The Author(s). 2018 Open Access This article is distributed under the terms of the Creative Commons Attribution 4.0 International License (http://creativecommons.org/licenses/by/4.0/), which permits unrestricted use, distribution, and reproduction in any medium, provided you give appropriate credit to the original author(s) and the source, provide a link to the Creative Commons license, and indicate if changes were made. The Creative Commons Public Domain Dedication waiver (http://creativecommons.org/publicdomain/zero/1.0/) applies to the data made available in this article, unless otherwise stated.

cow housing, feeding, and management have increased milk yield by an additional $2503 \mathrm{~kg}$, with the combined effect of genetic selection and management resulting in an approximate doubling of milk yield in five decades. While highly successful, the genes and physiological processes which have been altered to facilitate such increases remain elusive. A notable exception is a binucleotide substitution in the diacylglycerol O-acyltransferase 1 (DGAT1) gene that causes a lysine to alanine substitution at position $232(K 232 A)[5,6]$. The alanine variant results in higher milk and protein yield, but is not economically advantageous in many markets because of a substantial correlated decline in milk-fat yield. DGAT1 is the largest quantitative trait 
locus (QTL) for milk, fat, and protein yield [7] reported to date.

More recently, with the development and application of the Illumina BovineSNP50 BeadChip [8] and subsequent BeadChips of varying marker density [9], dairy cattle selection programs have incorporated genomic predictions facilitated by marker genotypes for thousands of loci spread across the genome [10]. Genomic analysis has largely confirmed the quantitative model of many small effects that cumulatively account for a high degree of variation [11], but understanding of how selection alters performance remains elusive. Collaborative efforts to sequence a large reference population of cattle from many breeds, such as the 1000 bull genomes project [12], will provide further insights into DNA sequence variation and potential impacts of such variation on cattle phenotypes [13], but increasing gene marker density beyond $\sim 50,000$ markers has not improved the accuracy of genomic predictions by a substantial degree to date [14].

While significant efforts have been expended studying associations of DNA sequence variation with cattle performance, epigenetic variations and their contribution to milk yield and cow health have received little attention and there are no known epigenetic-QTL for milk yield. Of particular interest to dairy cattle breeders would be linkages between epigenetic variation and the health of cows. Selection for yield [15], higher levels of confinement, and shifts in housing and feeding stratagies [16] are unfavorably associated with cow health and wellbeing. Emerging evidence from humans has shown a role for epigenetics in important cattle diseases [17] such as metabolic failure [18, 19], respiratory infection [20], and lymphoma [21], but this has not been studied extensively in livestock species.

Shifts in DNA methylation are plausible molecular mechanisms for phenotypic change in milk yield and cow health due to selection and management [22], but the cattle methylome must be more completely described before such effects can be determined [23]. The objective of this study was to identify genome-wide DNA methylation patterns in Holstein peripheral blood mononuclear cells (PBMC) to provide a resource for further investigation into causes of phenotypic variation in high milk-yield dairy cows.

\section{Results \\ Animals}

Blood was collected from the coccygeal vein (tail vein) of 6 lactating Holstein dairy cows from four commercial Pennsylvania dairy farms. Results from their official genomic evaluation for milk, fat and protein yields plus phenotypic records for yield during the parity of DNA sampling are reported in Table 1.

Four cows represented case-control pairs and the protocol for selecting these pairs is described in the methods. All cows were housed in tie-stalls and fed a total-mixed ration that was top-dressed according to the cow's nutritional requirements. The intermediate milk yield cows were from farms that allowed pasture access for lactating cows during the summer, whereas the case-control cows remained confined during lactation. The high milk yield cows averaged $21,052 \mathrm{~kg}$ of milk during the parity of blood sampling, whereas control cows averaged 14,677 $\mathrm{kg}$ of milk. The 2 remaining cows were selected from separate herds to increase the number of cows and environments represented and had milk yield that was intermediate $(16,236 \mathrm{~kg})$ to the high and control cows. Identifying cows with extremely high milk yield required that we sample cows from generally well-managed herds; consequently, our control cows had milk yield that was somewhat higher than the national average of 12,087 kg for Holsteins [3].

\section{General methylation patterns in the genome of Holstein cows}

Clean reads were aligned to btau4.0 with an average across cows of $64 \%$ of clean reads uniquely mapped; of these $60 \%$ were mapped to repetitive elements and $40 \%$ to unique sequences. The proportion of unique reads mapped to non-repetitive elements near gene bodies is shown in Fig. 1. These reads were most likely to be mapped to introns $(15.0 \%)$; the combined proportion for

Table 1 Official genomic estimated breeding values, observed yields, age, and parity of DNA sampling

\begin{tabular}{|c|c|c|c|c|c|c|c|c|c|}
\hline Cow & Description & $\begin{array}{l}\text { gEBV } \\
\text { Milk (kg) }\end{array}$ & $\begin{array}{l}\text { gEBV } \\
\text { Fat (kg) }\end{array}$ & $\begin{array}{l}\text { gEBV } \\
\text { Protein (kg) }\end{array}$ & Milk (kg) & Fat (kg) & Protein (kg) & $\begin{array}{l}\text { Age } \\
\text { (mo.) }\end{array}$ & Parity \\
\hline $\mathrm{F} 1 \mathrm{H}$ & Farm 1 Case & 624 & 8 & 5 & 23,105 & 1028 & 674 & 61 & 2 \\
\hline F1L & Farm 1 Control & -1316 & -26 & -25 & 15,798 & 498 & 477 & 71 & 2 \\
\hline $\mathrm{F} 2 \mathrm{H}$ & Farm 2 Case & -967 & -12 & -34 & 19,000 & 764 & 533 & 84 & 4 \\
\hline $\mathrm{F} 2 \mathrm{~L}$ & Farm 2 Control & -740 & -56 & -34 & 13,556 & 454 & 369 & 85 & 5 \\
\hline F3l & Farm 3 & -907 & -57 & -40 & 16,094 & 576 & 443 & 69 & 4 \\
\hline F4l & Farm 4 & 171 & -15 & -6 & 16,379 & 685 & 463 & 116 & 4 \\
\hline
\end{tabular}

$\mathrm{gEBV}=$ genomic estimated breeding values provided from the Council on Dairy Cattle Breeding; positive values indicate that cows are expected to produce more milk, fat or protein than the average cow born in 2010 and negative values indicate cows expected to produce less. Milk, fat and protein yields (kg) are standardized to a constant 305 day parity length and age [54] 


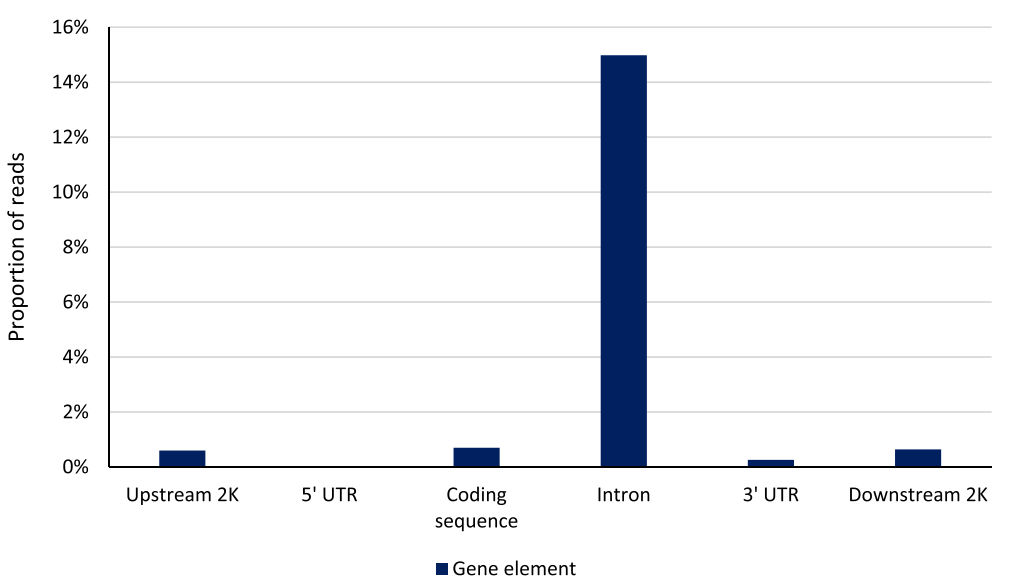

Fig. 1 Proportion of reads mapped to gene elements. Percent of reads mapped $2 \mathrm{~kb}$ upstream of the transcription start site, in the $5^{\prime}$-untranslated region, in the protein coding sequence, in introns, in the $3^{\prime}$-untranslated region, and $2 \mathrm{~kb}$ downstream of the transcription termination site

the other gene elements totaled $2.2 \%$ with the $5^{\prime}$-untranslated region accounting for only $0.03 \%$ of reads. Figure 2 reports the proportion of repetitive element reads, which were primarily mapped to satellite (36.4\%), SINE (29.1\%), and LINE (23.7\%) regions. The majority (78.4\%) of CpG sites were sequenced at least once and $19.8 \%$ were sequenced at a depth of 5 or greater. In contrast, the genome-wide sequencing depths were $28.5 \%$ and $3.7 \%$ for depths of $\geq 1$ or $\geq 5$, respectively. CHG ( $\mathrm{H}$ $=\mathrm{A}, \mathrm{C}$, or $\mathrm{T})$ sites $(43.5 \%)$ were more likely to be sequenced at least once than $\mathrm{CHH}$ sites $(38.5 \%)$. The proportion of reads mapped by $\mathrm{CpG}$ density is shown in Fig. 3. Approximately two-thirds of reads were mapped to regions that contained between 5 and $20 \mathrm{CpG}$ per kb.

The proportion of the genome covered by methylation peaks ranged from 7.38\% (cow F4I) to $10.52 \%$ (cow F1L) with an average of $8.43 \%$. Average peak size ranged from 735 bp to 1080 bp for cows F3I and F1L, respectively. The proportion of gene element types covered by a peak is displayed in Fig. 4 . The majority of coding sequence (86.8\%) and $5^{\prime}$-untranslated region sequence $(76.8 \%)$ resided within a methylation peak. In contrast, only $11.6 \%$ of intronic sequence was covered by a peak. Coordinates for peak regions are reported for each cow in Additional file 1: Table S1, Additional file 2: Table S2, Additional file 3: Table S3, Additional file 4: Table S4, Additional file 5: Table S5, and Additional file 6: Table S6.

\section{Geometric mean reads (GMR)}

Following a visual inspection of results [24] and considering preliminary descriptive statistics, it was clear that reads were not normally distributed across cows or genomic regions. Skewed distributions were not unexpected as MeDIP enrichment preferentially targets GC rich regions because they are highly methylated and because of the existence of hypermethyated sites $[25,26]$. Bias can

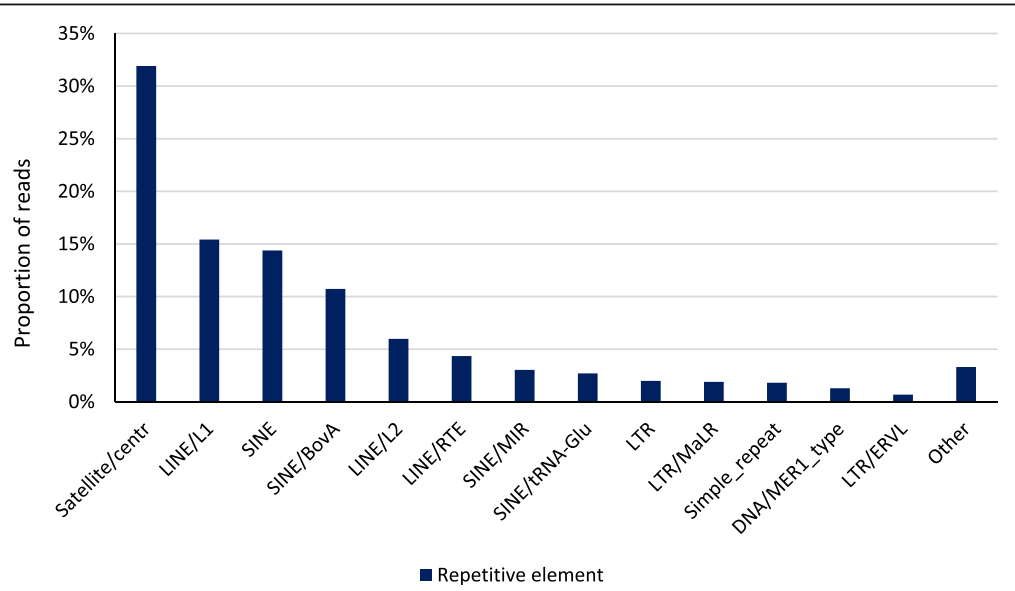

Fig. 2 Proportion of repetitive element reads mapped to each different element. Percent of repetitive element reads mapped to satellite/ centromeric, LINE, SINE, long terminal repeats, and other types of repetitive DNA sequence 


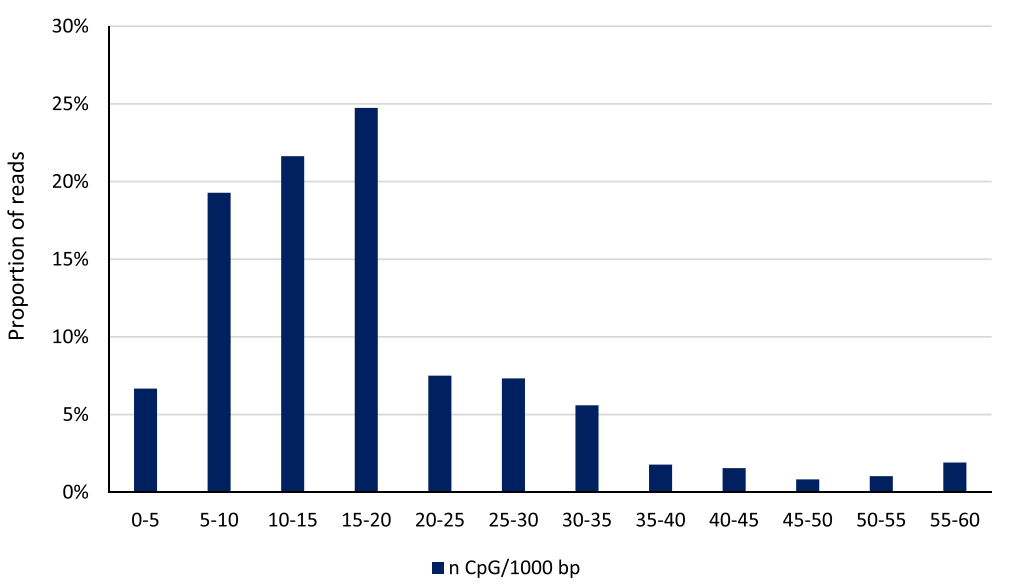

Fig. 3 Proportion of reads in relationship to the number of $\mathrm{CpG}$ sites per kb. The percentage of reads mapped to genomic regions by the relative density of $\mathrm{CpG}$ sites per $\mathrm{kb}$

also be introduced during library construction, particularly during PCR amplification of GC rich fragments [26]. Therefore, we derived a geometric mean reads (GMR) to describe general methylation patterns for this group of animals which is described in the methods. Figure 5 demonstrates alignment reads from two regions of the same length ( $\sim 860 \mathrm{bp})$. Figure 5 a demonstrates a region where two cows have a large number of reads, whereas Fig. 5b demonstrates a region with similar reads for all cows. The average normalized reads count (NRC) across these six cows was higher for the more variable region (0.32) than for the less variable region $(0.28)$ despite four of the cows having more reads in the second region. The average GMR ( $\mu$ GMR) reflects that the majority of cows had more reads for the less variable region $(\mu \mathrm{GMR}=1.61)$ than for the region with high variance $(\mu \mathrm{GMR}=1.44)$.
GMR of 13,677 unique Bos taurus protein coding genes downloaded from Ensembl [27] with completed coding sequence start and end coordinates were analyzed in the present study. $\mu \mathrm{GMR}$ for $1 \mathrm{~kb}$ upstream; first, middle and last exons; first and last intron; and $1 \mathrm{~kb}$ downstream are presented in Fig. 6 for these genes. Reads were generally low in the upstream region with the nadir $\mu$ GMR occurring at $95 \mathrm{bp}$ upstream of the TSS. $\mu$ GMR was lower in the first exon than in later exons, particularly in the first half of initial exons. The middle exon tended to be most highly methylated, whereas the last exons were generally highly methylated at the beginning of the exon and had lower methylation in the second half of the exon. $\mu$ GMR was highest for introns near the intron-exon junctions and were lowest in the middle and declined downstream as the distance from the gene increased.

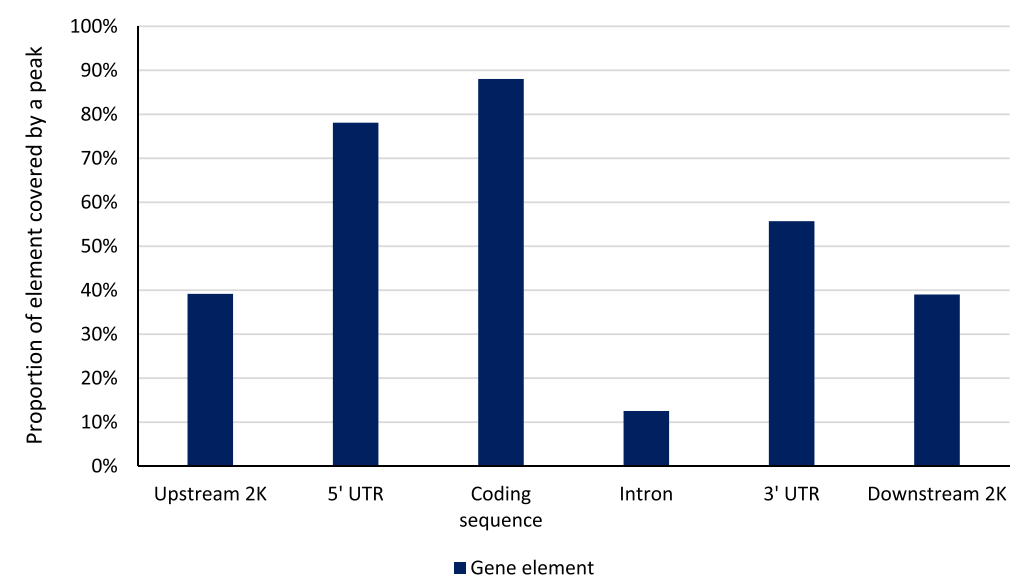

Fig. 4 Proportion of genomic elements covered by a methylation peak. The percentage of total length for each gene element covered by a DNA methylation peak divided by the total length of each element in the genome for $2 \mathrm{~kb}$ upstream of the transcription start site, the $5^{\prime}$-untranslated region, the protein coding sequence, introns, the $3^{\prime}$-untranslated region, and $2 \mathrm{~kb}$ downstream of the transcription termination site 


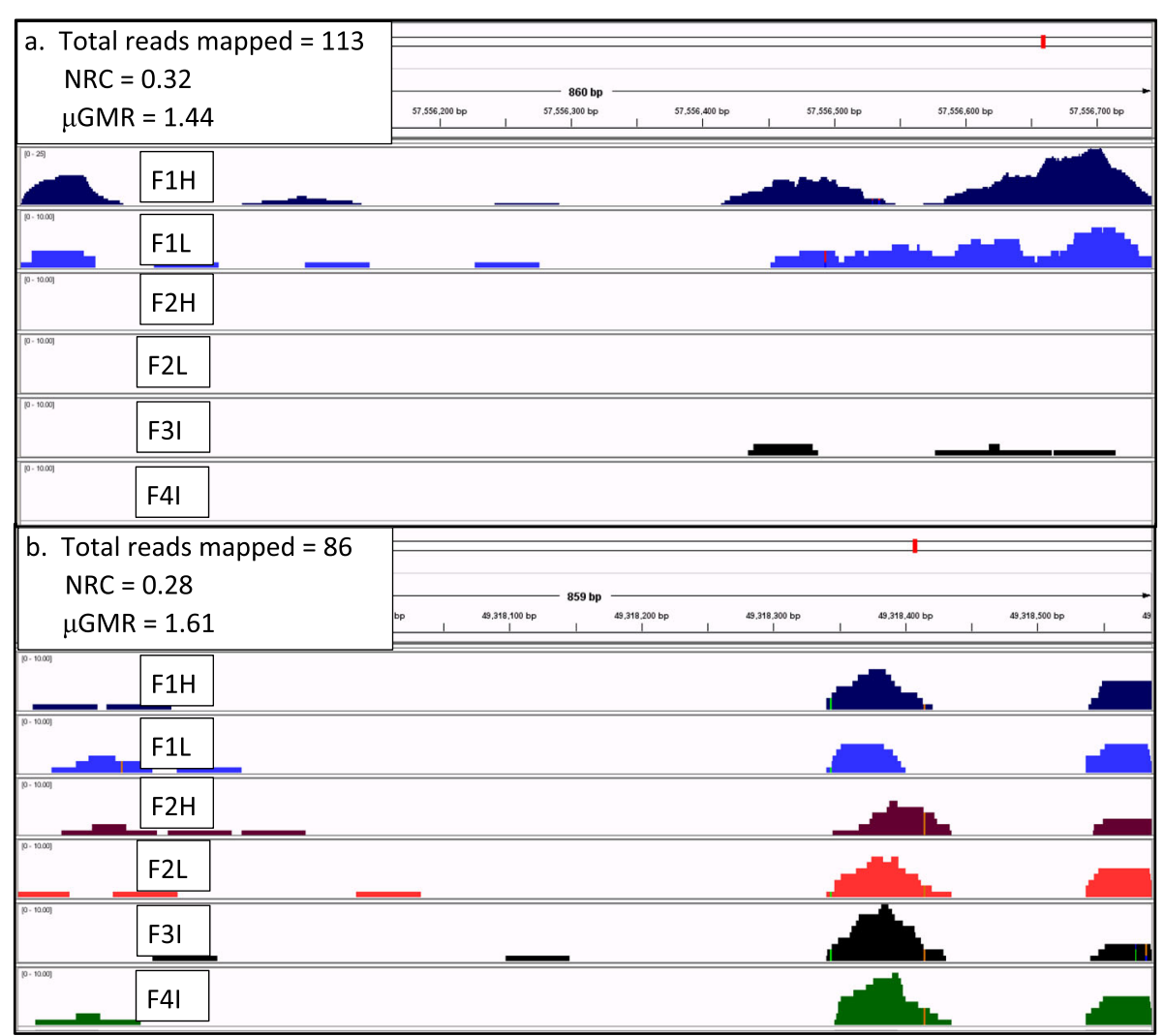

Fig. 5 Average normalized reads count versus geometric means reads for two example regions. Individual cow alignments [23] for two 860 bp regions. Panel $\mathbf{a}$. demonstrates alignments for a region with high variability among cows on BTA19:57,555,882-57,556,746 and that is a significant environmental DMR, whereas panel $\mathbf{b}$. demonstrates alignments for a region with minimal variation on BTA24:49,317,728-49,318,588. The identification of the cow corresponding to each panel is provided along with the total number of reads mapped to the region across all cows, the NRC, and $\mu G M R$

\section{Genome wide methylation patterns}

$\mu \mathrm{GMR}$ in non-overlapping $10 \mathrm{~kb}$ windows was determined to evaluate genome wide methylation patterns of uniquely mapped reads for all chromosomes (except for the Y chromosome) (see Additional file 7: Figure S1).
Visually, there appeared to be fewer reads mapped to the centromeric ends of many chromosomes than mapped to the remainder of the chromosome. $\mu$ GMR in the first $500 \mathrm{~kb}$ of the centromeric end (average of 0.81 \pm 0.68 across all chromosomes) were less $(P<0.001)$

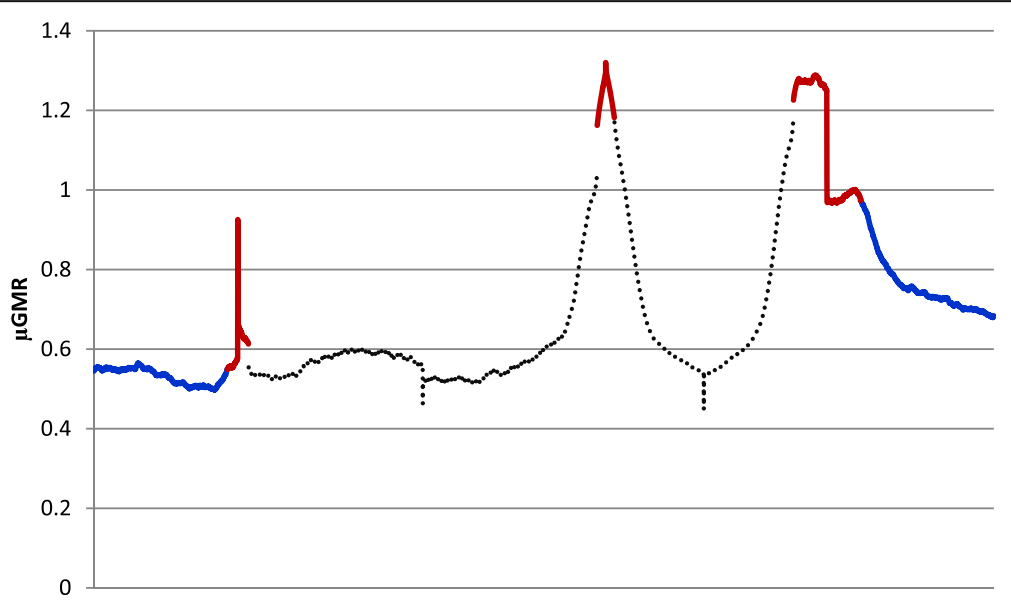

Fig. 6 Average geometric means reads across 13,677 genes. Average geometric means reads ( $\mu$ GMR) 1 kb upstream and 1 kb downsteam (blue); first, middle, and last exons (red); and first and last introns (black) for 13,677 bovine genes 
than the last $500 \mathrm{~kb}$ of the distal end (average of $1.06 \pm$ 1.00) and middle of the chromosomes (average of $1.07 \pm$ 0.51 ). These $\mu$ GMR only represented uniquely mapped reads and not repetitive satellite sequences which would be expected to concentrate at the centromeric ends of the chromosome. $\mu \mathrm{GMR}$ for the distal end and middle of the chromosomes were not different, but $\mu$ GMR was significantly less variable for middle of the chromosomes than for the centromeric and distal ends $(P<0.001)$. There were also large differences among chromosomes with the lowest for the $\mathrm{X}$ chromosome $(\mu \mathrm{GMR}=0.64$ ) and Bos taurus autosome (BTA) $6 \quad(\mu \mathrm{GMR}=1.00)$, whereas BTA19 $(\mu \mathrm{GMR}=1.25)$ and BTA27 $(\mu \mathrm{GMR}=$ 1.26) had the highest rates. The Spearman rank correlation between $\mu \mathrm{GMR}$ across $1 \mathrm{Mb}$ windows with the number of protein coding genes in the window was 0.39 $(P<0.0001)$, indicating that higher gene density was associated with higher $\mu$ GMR.

\section{Partially methylated domains (PMD)}

Genomic regions with suppressed levels of methylation have been described as PMD [28], and such regions were apparent when evaluating $\mu \mathrm{GMR}$ across $10-\mathrm{kb}$ windows. We used a permutation test to empirically identify PMD. There were 4725 PMD covering $511.2 \mathrm{Mb}(\sim 19 \%)$ of the genome identified, with $16 \%$ of autosomes and $66 \%$ of the $\mathrm{X}$ chromosome falling within a PMD. The largest single PMD was from 74.4 to $79.7 \mathrm{Mb}$ on the $\mathrm{X}$ chromosome, whereas the largest autosomal PMD stretched from 72.5 to $76.7 \mathrm{Mb}$ on BTA12.

There were 1153 genes located in significant PMD, including 898 autosomal genes and 255 on the X chromosome. This represented $6.8 \%$ of the 13,156 autosomal genes and $48.9 \%$ of the $\mathrm{X}$ genes. The PMD genes were submitted to DAVID [29, 30] for functional evaluation with 1021 matching known genes. There were 29 significant (FDR adjusted $P<0.05$ ) functional annotation charts that are reported in Additional file 8: Table S7. Of those, 14 were part of an annotation cluster with an enrichment score of 31.91. The annotation charts for genes in that cluster are reported in Table 2 and encompassed 224 total genes, including 101 genes belonging to the $\mathrm{Ol}$ factory Transduction KEGG pathway [31].

\section{Putative differentially methylated regions (DMR)}

There were 72 DMR with a significant false discovery rate (FDR; $P<0.05$ ) identified by determining the fold change in a standardized number of reads in case versus control cows as described in the methods. The chromosome, starting position and ending position of the DMR are provided in Additional file 9: Table S8. An additional 252 environment specific DMR (Additional file 10: Table S9) were identified with 229 associated with farm 1, 24 associated with farm 2, and 1 that was associated with both herds. There were 2 regions that were identified as both case-control DMR and environmental DMR.

Additional file 11: Figure S2 (case-control) and Additional file 12: Figure S3 (environmental) show the most significant DMR within their broader genomic region of up to $40 \mathrm{~kb}$ from genomic elements that included known protein coding genes, pseudogenes, uncharacterized ncRNAs, and spliceosomal RNAs. In some instances, multiple putative DMR are located in close proximity. Additionally, the reads mapped to the third most significant environmental DMR represented the region with high variability in Fig. 5a. Genes (108) that

Table 2 Significant functional annotation charts [29, 30] from the most highly enriched cluster for genes located within partially methylated domains

\begin{tabular}{|c|c|c|c|c|}
\hline Category & Term & No. of genes & Fold Change & FDR \\
\hline GOTERM_MF_DIRECT & olfactory receptor activity & 112 & 4.4 & $5.70 \mathrm{E}-41$ \\
\hline INTERPRO & Olfactory receptor & 112 & 4.4 & $5.00 \mathrm{E}-40$ \\
\hline UP_KEYWORDS & Olfaction & 112 & 4.4 & $6.20 \mathrm{E}-40$ \\
\hline UP_KEYWORDS & Sensory transduction & 117 & 3.9 & 4.40E-37 \\
\hline GOTERM_MF_DIRECT & G-protein coupled receptor activity & 119 & 3.5 & $2.50 \mathrm{E}-33$ \\
\hline KEGG_PATHWAY & Olfactory transduction & 101 & 3.8 & $5.20 \mathrm{E}-32$ \\
\hline UP_KEYWORDS & G-protein coupled receptor & 133 & 3.2 & $8.50 \mathrm{E}-32$ \\
\hline INTERPRO & GPCR, rhodopsin-like, 7TM & 134 & 3.2 & $9.60 \mathrm{E}-32$ \\
\hline INTERPRO & G protein-coupled receptor, rhodopsin-like & 133 & 3.2 & $9.80 \mathrm{E}-32$ \\
\hline UP_KEYWORDS & Transducer & 134 & 3.0 & $3.10 \mathrm{E}-29$ \\
\hline GOTERM_BP_DIRECT & G-protein coupled receptor signaling pathway & 93 & 3.4 & $9.30 \mathrm{E}-24$ \\
\hline UP_KEYWORDS & Receptor & 151 & 2.3 & 4.60E-21 \\
\hline UP_KEYWORDS & Cell membrane & 143 & 1.9 & $5.30 \mathrm{E}-12$ \\
\hline GOTERM_CC_DIRECT & plasma membrane & 172 & 1.6 & $5.00 \mathrm{E}-08$ \\
\hline
\end{tabular}


were within $5 \mathrm{~kb}$ of a DMR were submitted to DAVID $[29,30]$ for functional evaluation with 96 matching known genes. Eight genes (listed Additional file 13: Table S10) were identified as part of two significant annotation charts of HOX (SMART) and homeobox (INTERPRO) with FDR adjusted $P<0.10$.

\section{Discussion}

Epigenetic descriptions in high yielding dairy cattle that have undergone intensive genetic selection can serve as a model to examine effects of selection on DNA methylation. We focused on DNA methylation in PBMC for this study because blood is accessible and farmers are unlikely to approve of invasive tissue sampling from the elite, high yielding cows that are of particular interest because they are population outliers. While blood is accessible, PBMC are also a mixed cell population which may make the epigenetic signal less clear; nevertheless, it was previously reported that $\mathrm{CpG}$ site variability in leukocytes could serve as an accurate predictor if CpG site variability in other tissues [32]. The large deviations of expected to actual milk, fat, and protein yield (Table 1) suggest that the differences in milk yield were not attributable to DNA sequence variation.

Many features of the cattle methylome reported here are shared with those from other species. The well described decline in promoter methylation [33, 34] (Fig. 2) was apparent. There is growing evidence that methylation is associated with alternative splicing across tissues [35] and that DNA methylation marks exon boundaries [36]. High levels of methylation across exons with a rapid decline of intronic methylation as distance from the exon-intron junction increased should be expected if methylation was a key exon marker. This was evident here with average methylation levels in the middle of introns as low as levels observed in the upstream region. Genes associated with the sensory perception of smell were highly enriched in human PMD [28]. We also found olfactory related genes to be enriched in PMD (Table 2), suggesting that PMD may be consistent across species. We noted a positive correlation between DNA methylation levels and gene density, which was previously reported for pigs [33] and in humans [37].

DNA methylation patterns from fetal and adult longissumus dorsi muscle were previously reported for Chinese Qinchuan cattle using a MeDIP-seq approach [34]. Average peak lengths were 974 (fetal) to 994 bp (adult) which are similar to our observations; a smaller proportion of the genome was reported to covered by a peak (4.4\% for fetal and $4.6 \%$ for adult muscle) than was observed in the current study. They also observed more peaks aligned to regions with 5 to $10 \mathrm{bp}$ per $\mathrm{kb}$, whereas we observed higher levels between 15 and $20 \mathrm{bp}$. Reads mapped to gene elements were most likely to be aligned to an intron in both fetal and adult muscle, as we observed in the current study (Fig. 1). However, this was the result of the long length of introns in comparison to other gene elements. In contrast, a very small percentage of reads aligned to the $5^{\prime}$-untranslated region, but approximately $90 \%$ of $5^{\prime}$-untranslated region and coding sequences had a methylation peak both fetal and adult muscle tissue which is similar to what we observed in PBMC.

Higher rates of DNA methylation are reported for most genes on the inactive $\mathrm{X}$ chromosome when compared with the active X-chromosome [38], resulting in higher X-chromosome DNA methylation rates for females than for males [33]. However, hypermethylation of the inactive $\mathrm{X}$ is concentrated on promotors of silenced genes and other features of the X-chromosome may result in lower chromosome wide DNA methylation levels. The frequency of CpG islands on the human $\mathrm{X}$ was reported to be half of the genome-wide frequency, gene density is low on the $\mathrm{X}$, and most $\mathrm{X}$ genes are relatively short [39].

We are unable to determine whether any of the DMR are directly responsible for phenotypic variation because a functional evaluation of epigenetic alterations is beyond the scope of this study and the transcriptomic data are not available for these individuals. For this reason, we refer to these regions as putative DMR. Nevertheless, there were regions that appear to harbor differential methylation and that have been previously associated with phenotypic variation in cattle. The putative DMR in the SECTM1 region (Additional file 11: Figure S2 k) is intriguing because of SECTM1 function and previously reported associations with cattle performance. Genes with roles in immune function are strong candidates for differential methylation in this study because we isolated DNA from PBMC. SECTM1 is highly expressed in leukocytes [40] and SECTM1 and CD7 are reported to be $I N F-\gamma$ induced co-stimulators of T-cell proliferation [41]. SECTM1 appears to have a role in in cattle immunity as there was a reported 2.73 fold increase in SECTM1 expression in Angus cattle that were resistant to parasitic infection than in those that were susceptible [42]. Humans are reported to have a single SECTM1 gene whereas there are multiple paralogs in cattle that have undergone positive selection [43]. The $60 S$ ribosomal protein $L 7$ pseudogene (Additional file 7: Figure S1f) was reported to have reduced expression in cattle with tuberculosis [44]. Additional regions have plausible relationships to differential methylation or immune function. For instance, the NLK pathway (Additional file 11: Figure S2c) is associated with transcriptional silencing via the methylation of PPARG target promoters at histone $H 3 K 9$ (http://www.uniprot.org/uniprot/Q9UBE8) [45], whereas three putative environmental 
DMR (Additional file 12: Figure S3a) are associated with FCGR2B which is associated with B-cell antibody production and immune complex phagocytosis (http://www.uniprot.org/uniprot/P31994) [45].

The functional annotation analysis indicated that homeobox genes were overrepresented in regions with DMR. Homeobox genes are reported to be differentially expressed in leukocytes [46]. However, caution is warranted when evaluating this pathway because of the relatively small number of genes [8] that were part of these functional charts.

\section{Conclusions}

Evaluating DNA methylation in populations that have undergone intense genetic selection may further our understanding of the role of DNA methylation in population change. We describe DNA methylation patterns for dairy cows with extreme phenotypes for milk yield and report the existence of putative DMR with plausible, but unverified, relationships to phenotypic and performance. This reference methylome for high producing Holstein cows provides a resource to more fully evaluate such relationships between variation in DNA methylation and phenotypic variation.

\section{Methods}

\section{Animals and blood samples}

Ten $\mathrm{mL}$ of whole blood was obtained from the coccygeal vein of 6 lactating Holstein dairy cows located on 4 commercial Pennsylvania dairy farms (Pennsylvania State Institutional Animal Care and Use Committee protocol number 28889). Following centrifugation, the buffy coat was extracted and stored $\left(-20^{\circ} \mathrm{C}\right)$ until DNA was extracted with a DNeasy ${ }^{\circ}$ Blood \& Tissue Kit (QIAGEN Sciences, Germantown, MD) per manufacturer instructions.

In order to identify commercial cows with extreme high milk yield phenotypes, we contacted 15 farms that participated in a milk testing program and that had herd average production approximately $50 \%$ greater than the average of Pennsylvania herds. The highest milk yielding cow from each herd and the poorest milk producing herd-mate of a similar age and parity were then selected and genotyped to generate genomic estimated breeding values (Table 1). Following genotyping, we selected two case-control pairs to maximize the likelihood that yield differences were due to epigenetic changes independent of DNA sequence variation. Additional potential case-control pairs were genotyped, but not selected for MeDIP-seq because yield deviations appeared to be explained largely by differences in genotype. The two intermediate production cows were also selected from the pool of high producing herds.

\section{MeDIP-seq and GMR}

MeDIP-seq and a bioinformatics analysis was conducted by BGI (Shenzhen, China). Library construction followed a previously described protocol [47] and consisted of genomic DNA fragmentation $(100-500$ bp by sonication), 3'-A overhang and ligation of sequencing adaptors (Illumina Pair-End DNA Sample Prep Kit), denaturing of double-stranded DNA, immunoprecipitation via 5-mC antibody, and PCR amplification and size selection (200-300 bp, including adaptor sequence). Both methylated DNA controls and unmethylated DNA controls were used with each DNA sample to validate the pulldown procedure. Approximately 100 million paired-end reads were generated for each cow and the 49 bp ends were sequenced using an Illumina HiSeq 2000 sequencer. Sample libraries were indexed to facilitate multiplexed sequencing per lane [48].

Raw reads were processed to remove those containing $5^{\prime}$ or $3^{\prime}$ adapter sequences, unknown or low quality bases. The remaining clean reads were aligned to btau4.0 (http:// hgdownload.cse.ucsc.edu/goldenPath/bosTau4/chromosomes) using the Short Oligonucleotide Analysis Package (v1 [49]). Whole genome peak scanning was conducted with MACS 1.4.0 (http://liulab.dfci.harvard.edu/MACS/) and only included reads with 2 or fewer mismatched bp. Peaks were aligned to btau4.0 and coordinates were then converted (https://genome.ucsc.edu/cgi-bin/hgLiftOver) to UMD 3.1 [50]. The analysis of repeats was conducted using RepeatMasker (http://www.repeatmasker.org) and repeat annotations were retrieved from the USCS database (http://hgdownload.cse.ucsc.edu/goldenPath/bosTau4/bigZips/bosTau4.fa.out.gz). CpG islands were regions of $\geq 200 \mathrm{bp}, \mathrm{G}+\mathrm{C}$ content $\geq 50 \%$, and the ratio of observed to expected CpG > 0.6 [51].

A second alignment to the current bovine assembly [50] was completed by Appistry, Inc. (St. Louis, MO, USA). The quality of reads in BAM files after alignment was evaluated with Picard (http://broadinstitute.github.io/picard/) and median quality scores ranged from 29 to 31 . The number of reads per nucleotide (NR) was extracted using the mpileup option of SAMtools [52] with a minimum mapping quality $\geq 15$. The GMR was determined as $G M R=\mathrm{e}^{\operatorname{mean}(\ln (\mathrm{NR}+1))}-\ln (2) / 6$ in SAS (v 9.4; SAS Institute Inc., Cary, NC). The +1 term was added so that the natural log could be derived for cows with no reads at a given nucleotide and the - $\ln (2) / 6$ partly removes the +1 term and sets $\mathrm{GMR}=1$ if a single cow has a single read at a nucleotide. GMR was set to 0 if all 6 cows had no reads at a given nucleotide. A normalized reads count was calculated for the regions depicted in Fig. 1 as $\left(R C^{*} 1,000,000\right) /(U R C)$ where $R C=$ the number of reads mapped to the region and URC = the total number of genome wide reads mapped to a unique sequence for each cow. 
We standardized exon and intron lengths so that general methylation patterns for exons, introns, and exon-intron junctions could be described as opposed to non-specific methylation levels across the gene body. Exon lengths were standardized to the median exon length, which were $159 \mathrm{bp}$ for first exons, $128 \mathrm{bp}$ for all middle exons, and $503 \mathrm{bp}$ for last exons. If there were more than $159 \mathrm{bp}$ for the first exon, the first $79 \mathrm{bp}$ were classified as nucleotides 1 to 79 , the last 79 bp were classified as 81 to 159 , and all others were classified as nucleotide 80. If there were fewer than $159 \mathrm{bp}$, the first $50 \%$ of nucleotides were associated with the first nucleotides of the exon, whereas the last $50 \%$ were associated with the last nucleotides of the exon. The same approach was used for the other exons and introns, with median intron lengths of $2616 \mathrm{bp}$ for first introns and $1343 \mathrm{bp}$ for last introns.

\section{Statistical analysis}

Statistical analyses were performed with SAS. Differences in $\mu$ GMR between the centromeric ends, middle, and distal ends of chromosomes were evaluated with the TTEST procedure. Likewise, tests of heterogeneous variance between the centromeric ends, middle, and distal ends of chromosomes were evaluated with the TTEST procedure.

\section{Identification of putative DMR}

There were $1,223,424$ regions with a length of $200 \mathrm{bp}$ to 4199 bp (1,186,289 autosomal and 37,135 X chromosome) with regions defined as a continuous stretch of nucleotides with reads present for one or more cows; regions that were less than $200 \mathrm{bp}$ apart were merged. Less than $1 \%$ of initial regions were $>4000 \mathrm{bp}$ in length and those large regions were split into regions of $4000 \mathrm{bp}$ with a restriction that the last segment had to be at least $200 \mathrm{bp}$ in length; this resulted in 1514 regions with a length between $4000 \mathrm{bp}$ and $4199 \mathrm{bp}$. The $\log _{2}$ of the total number of reads per region was determined for each cow and then standardized within cow to a mean of 100 and standard deviation of 5 . We derived a relative standardized fold change to identify DMR for case versus control cows because t-tests identified regions with low variation among cows as significant even if the differences between case and controls were minimal. We used a standardized change because cows may have zero reads in a region preventing direct calculation of the ratio between case and control cows.

A permutation test was conducted to determine expectations for case-control and environmental distributions. Reads from a randomly selected region were drawn and then the two cows from farm 1 (F1H and F1L) were jointly assigned to either herd environment 1 , herd environment 2, or herd environment 3 at random.
Cows from farm 2 were then randomly assigned to one of the two remaining herd environments, with cows F3I and F4I assigned to the final environment. This resulted in 6 possible herd environment combinations. Within each herd environment, one cow was randomly assigned to be the high yield case cow, with the second cow from the environment serving as the control. This yielded 8 possible case-control combinations within each herd environment, for a total of 48 randomly assigned scenarios which are shown in Additional file 14: Table S11. This process was repeated $58,724,352$ times, which was a rate of 48 samples per region with replacement. The actual number of times a region was randomly drawn ranged from 19 to 85 .

The ratio of standardized reads for the randomly assigned case cow to the randomly assigned control was determined for herd environment 1 and herd environment 2 , and the mean of those two ratios determined for each permutation. The ratio was not determined for the third herd environment because only data from two of the farms (farm 1 and farm 2) were case-control pairs. The mean (1.000) and standard deviation (0.0198) were then applied to the mean ratio of the two actual case-control pairs to determine the $P$-Value for the observed fold changes. P-Values were multiplied by 2 to account for the two-tailed aspect of the test. The $P$-Values from all regions were then evaluated with the MULTTEST procedure of SAS to derive the genome-wide FDR.

To identify putative environment specific DMR, we first derived the mean of standardized reads for cows randomly assigned to herd environment 1 , herd environment 2 , and herd environment 3 . The ratio of mean standardized reads for a single herd environment to the mean of standardized reads for all other cows was then derived for each permutation. The resulting mean (1.000) and standard deviation (0.0187) were then used to derive P-Values for ratios for farm 1 and farm 2 . While the intermediate milk yield cows (F3I and F4I) were used to derive permutations, they were not from the same herd so were not considered when identifying putative environment specific DMR as at least two observations per environment are required [53]. A single dataset that contained P-Values from each herd comparison and from all regions (3,670,272 P-Values) was then evaluated with the MULTTEST procedure of SAS to derive the genome-wide FDR.

Genes located within $5 \mathrm{~kb}$ of a DMR were submitted to DAVID [23] for functional evaluation with a medium classification stringency. There were 53 genes that fell within this range of a DMR and with a FDR adjusted $P$ $<0.05$. Therefore, we relaxed the FDR adjusted $P$-Value threshold to $P<0.10$ which increased the number of genes to 108 . The background genes were 13,677 unique 
bovine protein coding genes downloaded from Ensembl [23] which represented the pool of genes that we could detect overlapping DMR; of those, 12,980 were successfully converted to DAVID id numbers. We assumed default values which included medium classification stringency, and with annotation to three functional categories (COG_ONTOLOGY, UP_KEYWORDS, UP_SEQ_FEATURE), three gene ontology terms (GOTERM_BP_DIRECT, GOTERM_CC_DIRECT, GOTERM_MF_DIRECT), one pathway (KEGG_PATHWAY), and three protein domains (INTERPRO, PIR_SUPERFAMILY, SMART).

\section{Identification and evaluation of PMD}

A permutation test was conducted to identify significant PMD. The genome was partitioned into non-overlapping $10 \mathrm{~kb}$ windows and the proportion of each window covered by the methylated regions described above was determined. A moving average of percent coverage for ten consecutive $10 \mathrm{~kb}$ windows was then determined. We drew ten windows at random (with replacement) with this process was repeated 1 million times. Based on the permutation test, the average percent coverage across ten consecutive $10 \mathrm{~kb}$ windows was expected to fall below $25 \%$ for $1 \%$ of $\geq 100 \mathrm{~kb}$ moving average windows if methylation levels in adjoining $10 \mathrm{~kb}$ windows are independent. Genomic regions with less than this amount were considered significant PMD at $P<0.01$. Genes located within a PMD were submitted to DAVID [23] for functional evaluation using the same background genes and defaults as described for DMR.

\section{Additional files}

Additional file 1: Table S1. Chromosome, start nucleotide, end nucleotide, length, summit, tags, $\log P$, and fold enrichment of significant peaks for Farm 1, case. DNA methylation peak information for Farm 1, case. (XLSX $14158 \mathrm{~kb})$

Additional file 2: Table S2. Chromosome, start nucleotide, end nucleotide, length, summit, tags, $\log P$, and fold enrichment of significant peaks for Farm 1, control. DNA methylation peak information for Farm 1, control. (XLSX $13541 \mathrm{~kb}$ )

Additional file 3: Table S3. Chromosome, start nucleotide, end nucleotide, length, summit, $\operatorname{tags}, \log P$, and fold enrichment of significant peaks for Farm 2, case. DNA methylation peak information for Farm 2, case. (XLSX $14201 \mathrm{~kb})$

Additional file 4: Table S4. Chromosome, start nucleotide, end nucleotide, length, summit, tags, $\log P$, and fold enrichment of significant peaks for Farm 2, control. DNA methylation peak information for Farm 2, control. (XLSX 13499 kb)

Additional file 5: Table S5. Chromosome, start nucleotide, end nucleotide, length, summit, tags, $\log P$, and fold enrichment of significant peaks for Farm 3. DNA methylation peak information for Farm 3. (XLSX $14317 \mathrm{~kb})$

Additional file 6: Table S6. Chromosome, start nucleotide, end nucleotide, length, summit, tags, $\log P$, and fold enrichment of significant peaks for Farm 4. DNA methylation peak information for Farm 4. (XLSX $13513 \mathrm{~kb})$
Additional file 7: Figure S1. Average geometric mean reads per nucleotide over $10 \mathrm{~kb}$ windows and the number of annotated genes per $\mathrm{Mb}$. Columns representing the $\mu \mathrm{GMR}$ of non-overlapping $10 \mathrm{~kb}$ windows for each chromosome and overlaid with an indication of the number of genes in each region. (PDF $1474 \mathrm{~kb}$ )

Additional file 8: Table S7. Functional annotation charts of genes located within partially methylated domains with the number of genes, fold enrichment, and FDR adjusted $P$-Value of the annotation chart. The functional category, term, number of genes, fold enrichment, and FDR adjusted $P$-Value of functional annotation charts with FDR adjusted $P$ $<0.05$. (DOCX $13 \mathrm{~kb}$ )

Additional file 9: Table S8. Location (chromosome, starting and ending nucleotide) of putative case-control differentially methylated regions, false discovery rate (FDR) adjusted $P$-value, and number of reads mapped to the region for case and control cows. The chromosome, starting nucleotide, ending nucleotide, FDR adjusted P-Value, and number of reads for each case and control cow for 72 regions with differential methylation. (DOCX $17 \mathrm{~kb})$

Additional file 10: Table S9. Location (chromosome, starting and ending nucleotide) of putative environmental differentially methylated regions, false discovery rate (FDR) adjusted $P$-value, and number of reads mapped to the region for all cows. The chromosome, starting nucleotide, ending nucleotide, FDR adjusted $P$-Value, and number of reads for each cow for 289 regions with differential methylation. (DOCX 29 kb)

Additional file 11: Figure S2. The location and surrounding genomic region for the top 11 most significant case-control differentially methylated regions. NCBI Genome Data Viewer of regions that harbor putative DMR with the location of each DMR and identification of nearby genes from NCBI Bos taurus Annotation Release 105, 2016-01-26. (PDF 294 kb)

Additional file 12: Figure S3. The location and surrounding genomic region for the top 10 most significant environmental differentially methylated regions. NCBI Genome Data Viewer of regions that harbor putative DMR with the location of each DMR and identification of nearby genes from NCBI Bos taurus Annotation Release 105, 2016-01-26. (PDF $119 \mathrm{~kb})$

Additional file 13: Table S10. Eight homeobox genes associated with a differentially methylated region (DMR) that were part of a single functional annotation chart with an FDR adjusted $P<0.05$. Ensembl gene ID, gene name, location of DMR, and location of gene TSS. (DOCX $12 \mathrm{~kb}$ )

Additional file 14: Table S11. 48 sampling scenarios implemented in permutations to identify significance thresholds for identifying differentially methylated regions. A list of the high and low cow selected for each herd for each of the 48 sampling strategies. (DOCX $13 \mathrm{~kb}$ )

\section{Abbreviations}

BTA : Bos taurus autosome; DMR: differentially methylated region; F1H: farm 1 high; F1L: farm 1 low; F2H: farm 2 high; F2L: farm 2 low; F3l: farm 3 intermediate; F4l: farm 4 intermediate; GMR : geometric mean reads; NR : number of reads per nucleotide; NRC : normalized reads count; PBMC : peripheral blood mononuclear cells; PMD : partially methylated domains; QTL: quantitative trait locus; RC : reads count; TSS : transcription start site; URC : unique reads count; $\mu G M R$ : average geometric mean reads

\section{Acknowledgements}

The authors thank the dairy farmers who allowed access to their cows and staff members at the Penn State Dairy Research and Teaching Center for their assistance. Charlene Barlieb, Darryl Blakey, Pranoy Mohapatra and Jennell Coulter are appreciated for data preparation and preliminary data description and analysis.

\section{Funding}

This work was funded by CSREES 2008-34437-19335. The funding body was not involved with the design of the study, collection, analysis, interpretation of data, or writing of the manuscript. 


\section{Availability of data and materials}

The datasets generated and/or analysed during the current study are available in the NCBI Sequence Read Archive repository \#SRP071938, https:// www.ncbi.n/m.nih.gov/bioproject/PRJNA310241.

\section{Authors' contributions}

CD conceived the trial, conducted data analysis, and was responsible for manuscript preparation. WL assisted with data analysis and interpretation, and with manuscript preparation. Both authors read and approved the final manuscript.

\section{Ethics approval and consent to participate}

Sampling was conducted under Pennsylvania State Institutional Animal Care and Use Committee (IACUC) number 28889. Commercial herd owners provided consent to include their cows in the study.

\section{Consent for publication}

Not applicable.

\section{Competing interests}

A US patent application titled "Genomic Regions with Epigenetic Variation that Contribute to Phenotypic Differences in Livestock" has been filed.

\section{Publisher's Note}

Springer Nature remains neutral with regard to jurisdictional claims in published maps and institutional affiliations.

Received: 11 July 2018 Accepted: 27 September 2018

Published online: 11 October 2018

\section{References}

1. Hernando-Herraez I, Garcia-Perez R, Sharp AJ, Marques-Bonet T. DNA methylation: insights into human evolution. PLoS Genet. 2015;11:e1005661. https://doi.org/10.1371/journal.pgen.1005661.

2. Schübeler D. Function and information content of DNA methylation. Nature. 2015;517:321-6. https://doi.org/10.1038/nature14192.

3. Norman HD, Walton LM, Dürr J. State and national standardized lactation averages by breed for cows calving in 2014. CDCB Research Report K2-14. https://queries.uscdcb.com/publish/dhi/dhi16/laall.shtml. Accessed February 1, 2018

4. Council on Dairy Cattle Breeding. Trend in Milk BV for Holstein or Red \& White. https://queries.uscdcb.com/eval/summary/trend.cfm?R_Menu=HO. m\#StartBody. Accessed February 1, 2018.

5. Riquet J, Coppieters W, Cambisano N, Arranz JJ, Berzi P, Davis SK, Grisart B, Farnir F, Karim L, Mni M, et al. Fine-mapping of quantitative trait loci by identity by descent in outbred populations: application to milk production in dairy cattle. Proc Natl Acad Sci U S A. 1999;96:9252-7.

6. Grisart B, Coppieters W, Farnir F, Karim L, Ford C, Berzi P, Cambisano N, Mni M, Reid S, Simon P, et al. Positional candidate cloning of a QTL in dairy cattle: identification of a missense mutation in the bovine DGAT1 gene with major effect on milk yield and composition. Genome Res. 2002;12:222-31.

7. Wiggans GR, Cole JB, Hubbard SM, Sonstegard TS. Genomic selection in dairy cattle: The USDA experience. Annu Rev Anim Biosci. 2017;5:309-27.

8. Matukumalli LK, Lawley CT, Schnabel RD, Taylor JF, Allan MF, Heaton MP O'Connell J, Moore SS, Smith TP, Sonstegard TS, et al. Development and characterization of a high density SNP genotyping assay for cattle. PLoS One. 2009:4(4):e5350.

9. Wiggans GR, Cooper TA, Van Tassell CP, Sonstegard TS, Simpson EB. Technical note: characteristics and use of the Illumina BovineLD and GeneSeek genomic profiler low-density bead chips for genomic evaluation. J Dairy Sci. 2013;96:1258-63. https://doi.org/10.3168/jds.2012-6192.

10. VanRaden PM, Van Tassell CP, Wiggans GR, Sonstegard TS, Schnabel RD, Taylor JF, Schenkel FS. Invited review: reliability of genomic predictions for north American Holstein bulls. J Dairy Sci. 2009;92:16-24. https://doi.org/10. 3168/jds.2008-1514.

11. Cole JB, VanRaden PM, O'Connell JR, Van Tassell CP, Sonstegard TS, Schnabel RD, Taylor JF, Wiggans GR. Distribution and location of genetic effects for dairy traits. J Dairy Sci. 2009;92:2931-46. https://doi.org/10.3168/ jds.2008-1762.

12. 1000 bull genomes project. http://www.1000bullgenomes.com/. Accessed September 7, 2017.
13. Daetwyler HD, Capitan A, Pausch H, Stothard P, van Binsbergen R, Brøndum RF, Liao X, Djari A, Rodriguez SC, Grohs C, et al. Whole-genome sequencing of 234 bulls facilitates mapping of monogenic and complex traits in cattle. Nat Genet. 2014;46:858-65. https://doi.org/10.1038/ng.3034

14. VanRaden PM, Null DJ, Sargolzaei M, Wiggans GR, Tooker ME, Cole JB, Sonstegard TS, Connor EE, Winters M, van Kaam JB, et al. Genomic imputation and evaluation using high-density Holstein genotypes. J Dairy Sci. 2013;96:668-78. https://doi.org/10.3168/jds.2012-5702.

15. Shook GE. Selection for disease resistance. J Dairy Sci. 1989;72:1349-62.

16. Dechow CD, Smith EA, Goodling RC. The effect of management system on mortality and other welfare indicators in Pennsylvania dairy herds. Animal Welfare. 2011;20:145-58.

17. McConnel CS, Garry FB, Hill AE, Lombard JE, Gould DH. Conceptual modeling of postmortem evaluation findings to describe dairy cow deaths. J Dairy Sci. 2010;93:373-86. https://doi.org/10.3168/jds.2009-2296.

18. Seki Y, Williams L, Vuguin PM, Charron MJ. Minireview: epigenetic programming of diabetes and obesity: animal models. Endocrinology. 2012; 153:1031-8. https://doi.org/10.1210/en.2011-1805.

19. Brumbaugh DE, Friedman JE. Developmental origins of nonalcoholic fatty liver disease. Pediatr Res. 2014;75:140-7. https://doi.org/10.1038/pr.2013.193.

20. Pérez-Novo CA, Bachert C. DNA methylation, bacteria and airway inflammation: latest insights. Curr Opin Allergy Clin Immunol. 2015;15:27-32. https://doi.org/10.1097/ACl.130.

21. Ansell SM. DNA methylation in lymphoma: an opportunity? Blood. 2015; 125(12):1848-9.

22. Ibeagha-Awemu EM, Zhao X. Epigenetic marks: regulators of livestock phenotypes and conceivable sources of missing variation in livestock improvement programs. Front Genet. 2015;6:302. https://doi.org/10.3389/fgene.2015.302.

23. Couldrey C, Cave V. Assessing DNA methylation levels in animals: choosing the right tool for the job. Anim Genet. 2014;45(Suppl 1):15-24. https://doi. org/10.1111/age.12186.

24. Robinson JT, Thorvaldsdóttir H, Winckler W, Guttman M, Lander ES, Getz G, Mesirov JP. Integrative genomics viewer. Nat Biotechnol. 2011;29:24-6. https://doi.org/10.1038/nbt.1754

25. Lienhard M, Grasse S, Rolff J, Frese S, Schirmer U, Becker M, Börno S, Timmermann B, Chavez L, Sültmann H, Leschber G, Fichtner I, Schweiger MR, Herwig R. QSEA-modelling of genome-wide DNA methylation from sequencing enrichment experiments. Nucleic Acids Res. 2017;45:e44. https:// doi.org/10.1093/nar/gkw1193.

26. Robinson MD, Stirzaker C, Statham AL, Coolen MW, Song JZ, Nair SS, Strbenac D, Speed TP, Clark SJ. Evaluation of affinity-based genome-wide DNA methylation data: effects of CpG density, amplification bias, and copy number variation. Genome Res. 2010;20(12):1719-29.

27. Flicek P, Ahmed I, Amode MR, Barrell D, Beal K, Brent S, Carvalho-Silva D, Clapham P, Coates G, Fairley S, et al. Ensembl 2013. Nucleic Acids Res. 2013; 41:D48-55. https://doi.org/10.1093/nar/gks1236.

28. Schroeder DI, Lott P, Korf I, LaSalle JM. Large-scale methylation domains mark a functional subset of neuronally expressed genes. Genome Res. 2011; 21:1583-91. https://doi.org/10.1101/gr.119131.110.

29. Huang da W, Sherman BT, Lempicki RA. Bioinformatics enrichment tools: paths toward the comprehensive functional analysis of large gene lists. Nucleic Acids Res. 2009;37(1):-13. https://doi.org/10.1093/nar/gkn923.

30. Huang da W, Sherman BT, Lempicki RA. Systematic and integrative analysis of large gene lists using DAVID bioinformatics resources. Nat Protoc. 2009;4: 44-57. https://doi.org/10.1038/nprot.2008.211.

31. Kanehisa M, Goto S. KEGG: Kyoto encyclopedia of genes and genomes. Nucleic Acids Res. 2000;28:27-30.

32. Ma B, Wilker EH, Willis-Owen SA, Byun HM, Wong KC, Motta V, Baccarelli AA, Schwartz J, Cookson WO, Khabbaz K, et al. Predicting DNA methylation level across human tissues. Nucleic Acids Res. 2014;42(6):3515-28.

33. Li M, Wu H, Luo Z, Xia Y, Guan J, Wang T, Gu Y, Chen L, Zhang K, Ma J, et al. An atlas of DNA methylomes in porcine adipose and muscle tissues. Nat Commun. 2012;3:850.

34. Huang YZ, Sun JJ, Zhang LZ, Li CJ, Womack JE, Li ZJ, Lan XY, Lei CZ, Zhang $\mathrm{CL}$, Zhao $\mathrm{X}$, et al. Genome-wide DNA methylation profiles and their relationships with mRNA and the microRNA transcriptome in bovine muscle tissue (Bos taurine). Sci Rep. 2014;4:6546

35. Wan J, Oliver VF, Zhu H, Zack DJ, Qian J, Merbs SL. Integrative analysis of tissue-specific methylation and alternative splicing identifies conserved transcription factor binding motifs. Nucleic Acids Res. 2013:41:8503-14. https://doi.org/10.1093/nar/gkt652. 
36. Gelfman S, Cohen N, Yearim A, Ast G. DNA-methylation effect on cotranscriptional splicing is dependent on GC architecture of the exonintron structure. Genome Res. 2013;23:789-99. https://doi.org/10.1101/gr. 143503.112.

37. Ball MP, Li JB, Gao Y, Lee JH, LeProust EM, Park IH, Xie B, Daley GQ, Church GM. Targeted and genome-scale strategies reveal gene-body methylation signatures in human cells. Nat Biotechnol. 2009;27(4):361-8.

38. Sharp AJ, Stathaki E, Migliavacca E, Brahmachary M, Montgomery SB, Dupre $Y$, Antonarakis SE. DNA methylation profiles of human active and inactive $X$ chromosomes. Genome Res. 2011;21(10):1592-600.

39. Ross MT, Grafham DV, Coffey AJ, Scherer S, McLay K, Muzny D, Platzer M, Howell GR, Burrows C, Bird CP, et al. The DNA sequence of the human X chromosome. Nature. 2005:434(7031):325-37.

40. Slentz-Kesler KA, Hale LP, Kaufman RE. Identification and characterization of K12 (SECTM1), a novel human gene that encodes a Golgi-associated protein with transmembrane and secreted isoforms. Genomics. 1998;47(3):327-40.

41. Wang T, Huang C, Lopez-Coral A, Slentz-Kesler KA, Xiao M, Wherry EJ, Kaufman RE. K12/SECTM1, an interferon-gamma regulated molecule, synergizes with CD28 to costimulate human T cell proliferation. J Leukoc Biol. 2012;91(3):449-59.

42. Li RW, Rinaldi M, Capuco AV. Characterization of the abomasal transcriptome for mechanisms of resistance to gastrointestinal nematodes in cattle. Vet Res. 2011:42:114

43. Larson JH, Kumar CG, Everts RE, Green CA. Everts-van der wind a, band MR, Lewin HA: discovery of eight novel divergent homologs expressed in cattle placenta. Physiol Genomics. 2006;25(3):405-13.

44. Churbanov A, Milligan B. Accurate diagnostics for bovine tuberculosis based on high-throughput sequencing. PLoS One. 2012;7(11):e50147.

45. The UniProt C. UniProt: the universal protein knowledgebase. Nucleic Acids Res. 2017:45(D1):D158-69.

46. Morgan $\mathrm{R}$, Whiting K. Differential expression of HOX genes upon activation of leukocyte sub-populations. Int J Hematol. 2008;87(3):246-9.

47. Li N, Ye M, Li Y, Yan Z, Butcher LM, Sun J, Han X, Chen Q, Zhang X, Wang J. Whole genome DNA methylation analysis based on high throughput sequencing technology. Methods. 2010;52(3):203-12.

48. Liu L, Hu N, Wang B, Chen M, Wang J, Tian Z, He Y, Lin D. A brief utilization report on the Illumina HiSeq 2000 sequencer. Mycology. 2011;2(3):169-91.

49. Li R, Li Y, Kristiansen K, Wang J. SOAP: short oligonucleotide alignment program. Bioinformatics. 2008;24(5):713-4.

50. Bos_taurus_UMD_3.1.1. http://www.ncbi.nlm.nih.gov/assembly/GCA_ 000003055.4. Accessed September 7, 2017

51. Gardiner-Garden M, Frommer M. CpG islands in vertebrate genomes. J Mol Biol. 1987;196(2):261-82.

52. Li H, Handsaker B, Wysoker A, Fennell T, Ruan J, Homer N, Marth G, Abecasis G. Durbin R; 1000 genome project data processing subgroup. The sequence alignment/map format and SAMtools. Bioinformatics. 2009;25:2078-9. https://doi.org/10.1093/bioinformatics/btp352.

53. Ziller MJ, Hansen KD, Meissner A, Aryee MJ. Coverage recommendations for methylation analysis by whole-genome bisulfite sequencing. Nat Methods. 2015;12(3):230-2. https://doi.org/10.1038/nmeth.3152

54. Schutz MM. Age-season standardization for yield traits. http://aipl.arsusda. gov/reference/ageseason.htm. Accessed September 7, 2017.

\section{Ready to submit your research? Choose BMC and benefit from:}

- fast, convenient online submission

- thorough peer review by experienced researchers in your field

- rapid publication on acceptance

- support for research data, including large and complex data types

- gold Open Access which fosters wider collaboration and increased citations

- maximum visibility for your research: over $100 \mathrm{M}$ website views per year

At $\mathrm{BMC}$, research is always in progress.

Learn more biomedcentral.com/submissions 